\title{
A comparative study of a modified filtering trabeculotomy and conventional trabeculectomy
}

\author{
This article was published in the following Dove Press journal: \\ Clinical Ophthalmology \\ 13 March 2015 \\ Number of times this article has been viewed
}

\author{
Juliane Matlach ${ }^{1, *}$ \\ Matthias Hipp ${ }^{1, *}$ \\ Martin Wagner ${ }^{2,3}$ \\ Peter U Heuschmann ${ }^{2,3}$ \\ Thomas Klink' \\ Franz Grehn' \\ 'Department of Ophthalmology, \\ ${ }^{2}$ Institute of Clinical Epidemiology \\ and Biometry, ${ }^{3}$ Comprehensive \\ Heart Failure Center, University \\ of Würzburg, Würzburg, Germany \\ *These authors contributed equally \\ to this work
}

Purpose: The objective of the study reported here was to evaluate the outcome of a modified filtering trabeculotomy (FTO) without iridectomy in open-angle glaucoma compared with that of conventional trabeculectomy (trab).

Patients and methods: Thirty eyes of 30 patients who underwent modified FTO were prospectively followed for 1 year and were compared with 87 conventional trab patients (87 eyes), matched for age and preoperative intraocular pressure (IOP). The FTO procedure consisted of a deep sclerectomy and trabeculotomy preserving the trabeculo-Descemet membrane, without iridectomy. Main outcome measures were complete success (IOP $<18 \mathrm{mmHg}$ and $>/=30 \%$ IOP reduction, without medication), IOP, visual acuity, medication, complications, and subsequent surgeries.

Results: In the conventional trab group, the median preoperative IOP was $23.0 \mathrm{mmHg}$ (interquartile range 20.0-27.0) with 3.0 (2.0-3.0) medications, compared with $23.0 \mathrm{mmHg}(20.0-27.0)$ and $3.0(2.8-4.0)$ in the modified FTO group. Median postoperative IOP at 12 months was $12.0 \mathrm{mmHg}(10.0-13.0)$ in the conventional trab and $11.0 \mathrm{mmHg}(8.0-14.0)$ in the modified FTO group $(P=0.3)$. The complete success rate at 1 year was $83.1 \%$ and $79.3 \%$ in the conventional trab group and modified FTO group, respectively $(P=0.8)$. The complications hypotony $(20.7 \%$, $24.1 \%)$, choroidal detachment $(2.3 \%, 10.3 \%)$, and bleb scarring $(17.2 \%, 13.8 \%)$, were present in the conventional trab group and modified FTO group, respectively.

Conclusion: The outcomes of reduced IOP and medications in the FTO group were not different to those in the conventional trab group over 1 year, but some complications were more often seen with the modified FTO technique. The new filtration trabeculotomy, however, has the advantage of avoiding iridectomy, thus reducing the risk of cataract formation, and may result in the development of more favorable blebs by controlling the flow over two resistance levels.

Keywords: glaucoma surgery, open-angle glaucoma, outflow resistance, iridectomy, trabeculectomy

\section{Introduction}

Modern filtration surgery aims at lowering intraocular pressure (IOP) by creating a new pathway for aqueous humor drainage while taking care of reducing complications associated with filtering blebs. Trabeculectomy (trab) has remained the most commonly performed glaucoma surgery since its first description by Cairns in $1968,{ }^{1}$ although bleb-related adverse events are frequently seen and necessitate intensive postoperative management and surgical interventions. Augmentation with antimetabolites such as mitomycin C (MMC) or 5-fluorouracil (5-FU) has been one of the major advances in glaucoma filtering surgery to reduce bleb failure. ${ }^{2}$ However, serious ocular complications such as hypotony, hypotony-related choroidal detachment, maculopathy, blebitis, or endophthalmitis are associated with the use of antimetabolites. ${ }^{3}$ Therefore, several modifications, combinations, and new techniques of trab have been described. ${ }^{4-23}$ Peripheral iridectomy (PI) is a part of filtration
Correspondence: Juliane Matlach Department of Ophthalmology, University of Würzburg, Josef-SchneiderStraße I I, 97080 Würzburg, Germany Tel +49 93I 20120600 Fax +49 931 20120490 Email juliane.matlach@outlook.com 
surgery routinely performed to avoid pupillary block and iris incarceration, but can also cause strong inflammatory response, hemorrhage, and cataract progression. ${ }^{24,25}$ Trab performed with PI is a safe procedure with a low incidence of postoperative inflammation and is as effective in lowering IOP as when performed without PI. ${ }^{26}$ The combination of trabeculotomy, as is often used in children with congenital glaucoma, and "sinusotomy" (partial excision of the outer scleral wall on Schlemm's canal) and non-penetrating trab, allowing for subconjunctival filtration and bleb formation, was previously described to improve the IOP-lowering effect of a filtering procedure while reducing hypotony-related complications..$^{27,28}$

The rationale for the filtering trabeculotomy (FTO) developed in the current study was to gradually lower IOP in two steps by allowing aqueous humor flow, first, via the opened Schlemm's canal and its ostia into the scleral lake, and, second, through the scleral flap into the subconjunctival space, thus providing a more gradual outflow. The second aim of this modified FTO was to avoid an iridectomy, possibly reducing anterior chamber inflammation, consecutive bleb failure, and accelerated cataract formation. The aim of the study reported here was to evaluate the outcome and safety of this modified FTO without PI compared with those of conventional trab in open-angle glaucoma over 1 year.

\section{Materials and methods Design}

The study protocol was approved by the local ethics committee of the University of Würzburg, Germany. Routinely collected general-practice data were compiled on patients in both surgical groups. All surgeries of trab and FTO were performed by a single surgeon (FG) between January 2007 and August 2011 (trab group) and September 2011 and March 2012 (FTO group). Patients undergoing FTO $(n=30)$ were prospectively followed for up to 1 year with visits scheduled at Days 1 and 7; Week 4; and Months 3, 6, and 12, with additional visits whenever clinically necessary. A control group of patients who underwent trab $(n=87)$ was selected by individualized matching for preoperative IOP and age (see the "Statistical methods" subsection).

Preoperatively, patient history was assessed, with data on type of glaucoma, previous ophthalmic laser or surgical treatment, and number of glaucoma medications obtained. The baseline ophthalmic examination involved visual acuity (VA) testing converted to the logarithm of the minimum angle of resolution ( $\log$ MAR), measurement of IOP using Goldmann applanation tonometry by a one-person technique, slit-lamp and fundus examination, and gonioscopy. At each follow-up visit, all patients had IOP and VA measurements taken and underwent slit-lamp biomicroscopy, fundus examination, bleb grading according to the Würzburg bleb classification score, ${ }^{29}$ and had at least one gonioscopy. Data were also collected on topical medication use, adverse events, and subsequent surgeries.

All patients were followed and examined at the Department of Ophthalmology, University of Würzburg. In case of missing data, ophthalmologists in private practice or clinics were asked to provide data on postoperative IOP and VA, topical anti-glaucomatous medication, complications, and further interventions.

\section{Inclusion and exclusion criteria}

One eye from each patient with primary or secondary (pseudo-exfoliative and pigmentary glaucoma) open-angle glaucoma who underwent conventional trab or FTO with MMC was included in the study. Reported baseline IOP values were calculated as the mean of at least three measurements taken at different time points of the preoperative day and - if available - on at least 2 different days within a month before surgery with topical medication but without any systemic anti-glaucoma medication.

Exclusion criteria were angle-closure, normal-tension, congenital, traumatic, neovascular, or uveitic glaucomas. Patients with prior penetrating or non-penetrating glaucoma surgeries, or more than two cyclodestructive (cyclophoto- or cyclocryocoagulation) or two laser trabeculoplasty procedures in the study eye were excluded.

\section{Outcomes}

The primary outcome was the rate of complete success according to the recommendations of the World Glaucoma Association, ${ }^{30}$ defined as an untreated IOP of $<18 \mathrm{mmHg}$ and IOP reduction of $30 \%$ or greater from treated baseline IOP. Secondary outcomes included qualified success (success criteria with or without medication), VA, use and number of glaucoma medications, postsurgical complications, and subsequent interventions.

\section{Surgical technique}

\section{Conventional trabeculectomy}

A corneal traction suture was placed at the 6 o'clock position ${ }^{31}$ and a fornix-based conjunctival flap was created. Blood vessels were cauterized using gentle diathermy. Different concentrations of MMC ( 0.1 to $0.5 \mathrm{mg} / \mathrm{mL})$ were used based on the individual risk of bleb scarring. The majority of patients 
were treated with MMC $0.2 \mathrm{mg} / \mathrm{mL}(\mathrm{n}=81,93.1 \%)$. MMC $0.1 \mathrm{mg} / \mathrm{mL}$ was used in three eyes (3.4\%), MMC $0.25 \mathrm{mg} / \mathrm{mL}$ in one eye (1.2\%), and MMC $0.5 \mathrm{mg} / \mathrm{mL}$ in two eyes $(2.3 \%)$ of the conventional trab group. Four MMC-soaked sponges measuring $2 \times 8 \mathrm{~mm}$ were placed under the conjunctiva for 3 minutes. The site of application was then washed with $30 \mathrm{~mL}$ of balanced salt solution. A rectangular half-thickness scleral flap of $4 \times 3 \mathrm{~mm}$ was created and a trab of $0.8 \times 2.0 \mathrm{~mm}$ was performed followed by a PI. The scleral flap was fixed with single 10.0 nylon sutures placed at both corners of the flap and additional sutures in between if necessary. The median number of single sutures was 4.0 (interquartile range [IQR] 4-4). A 10.0 nylon mattress suture was used to close the conjunctiva at the limbus.

\section{Modified filtering trabeculotomy}

A fornix-based conjunctival flap was created as described in the "Conventional trabeculectomy" subsection. In all cases, MMC $0.2 \mathrm{mg} / \mathrm{mL}$ was used for 3 minutes to prevent bleb scarring. A half-thickness $4 \times 4 \mathrm{~mm}$ scleral flap was dissected. A second, deep, posteriorly directed, tongue-shaped scleral flap was formed and its base advanced into clear cornea. This approach provided a safe and reproducible unroofing of Schlemm's canal and direct access to the ostia of Schlemm's canal on both sides. The deep flap was removed at its base and Schlemm's canal was gently dilated with viscoelastics (sodium hyaluronate 1.4\%; Healon ${ }^{\circledR}$, Abbott Medical Optics Inc, Santa Ana, CA, USA). Then, a trabeculotomy with the Mackensen trabeculotomy probe was performed on both sides to give the aqueous humor access to the intrascleral space through the opened Schlemm's canal. During trabeculotomy, meticulous care was taken to avoid breaking the trabeculo-Descemet window. Aqueous outflow was therefore controlled by the ostia of Schlemm's canal on this level. No PI was performed, as there was no direct opening into the anterior chamber.

The aim of this FTO was to lower IOP in two steps: (1) outflow of aqueous humor through the opened Schlemm's canal via the ostia into the scleral space as a first level of resistance and (2) outflow through the scleral flap under the conjunctiva as a second level of resistance. The intrascleral space was protected by hyaluronic acid separation to avoid the healing of the inner flap surface to the bottom of the scleral bed. An iridectomy was not needed due to the preservation of the inner wall of Schlemm's canal. The superficial scleral flap was secured with single $10 / 0$ nylon sutures. The median number of flap sutures was 4.0 (IQR 4-6). Finally, a 10.0 nylon running mattress suture was placed to
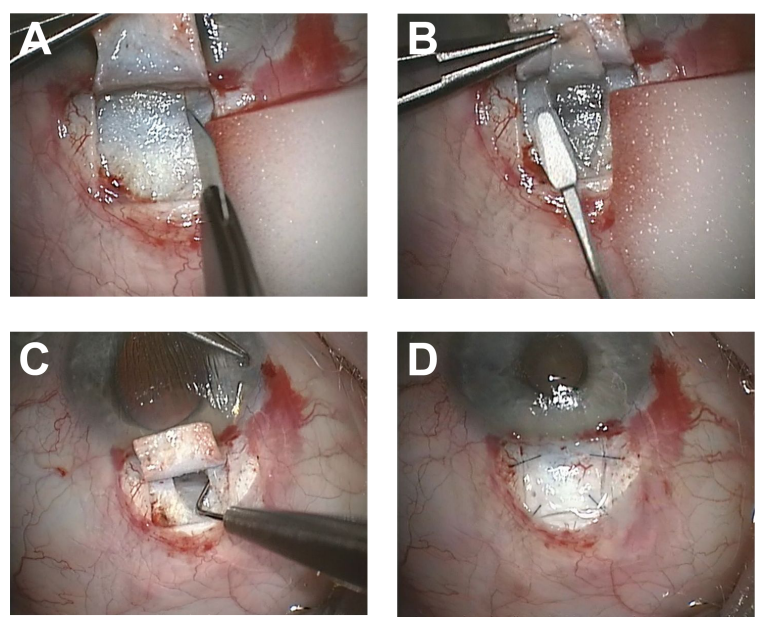

Figure I Surgical technique of modified filtering trabeculotomy. Notes: (A) A rectangular half-thickness scleral flap is created. (B) A deeper tongueshaped scleral flap is formed to allow access to Schlemm's canal. (C) A trabeculotomy is performed to both sides. (D) The superficial scleral flap is secured with single sutures after the deeper flap is removed.

close the conjunctiva (Figure 1A-D). Video S1 demonstrates the surgical technique of FTO surgery.

\section{Postoperative management}

Anti-glaucomatous medication was discontinued after surgery. All patients in both groups received a standardized postoperative medical therapy that entailed prednisolone acetate eye drops either every 1 or 2 hours for 1 week, tapering off over 6 to 8 weeks. Antibiotic eye drops (gentamicin three times per day) and cycloplegic eye drops (atropine twice per day) were given for 1 to 2 weeks. Early postoperative bleb management involved laser suture lysis of scleral flap sutures in case of elevated IOP and flat blebs that inflate after bleb massage; application of 5-FU bleb injections; intensified topical steroid application; and further surgical interventions, such as bleb needling or scleral flap revision for encapsulated blebs, according to the recommendations proposed by Marquardt et $\mathrm{l}^{132}$ of intensive postoperative care after trab. Bleb massage was rarely performed after FTO, as it is obviously less effective with the two-level outflow resistance.

\section{Statistical methods}

Statistical analyses were performed using SPSS (v 21.0; IBM Corporation, Armonk, NY, USA) and SAS (v 9.2; SAS Institute Inc, Cary, NC, USA) software. $P$-values of $<0.05$ were considered statistically significant. A group of $n=144$ patients who underwent open-angle glaucoma surgery performed by one surgeon (FG) between January 2007 and August 2011 served as the population of potential controls for the $\mathrm{n}=30$ individuals treated with FTO. A total of 90 patients (ratio 1:3, FTO:trab) were selected, using the 
“\%match" SAS macro, pair-matching for preoperative IOP (range $\pm 3 \mathrm{mmHg}$ ), and age (range \pm 8 years). Subsequently, three patients had to be excluded due to violation of inclusion criteria, thus leaving $n=87$ patients in the control group.

Baseline characteristics and efficacy and safety outcome information on FTO versus trab were compared by Student's $t$-test and the Mann-Whitney $U$-test (continuous variables according to normal distribution), and the $\chi^{2}$ test and Fisher's exact test; mean \pm standard deviation, median (IQR), and proportions are presented, as appropriate. Time-to-failure was analyzed by Kaplan-Meier cumulative incidence curves. Furthermore, outcome measures were analyzed by conditional exact logistic regression and conditional linear regression (maximum likelihood), considering individualized matching. Odds ratios, $\beta$-coefficients with respective $95 \%$ confidence intervals or $P$-values are reported.

\section{Results}

Thirty eyes of 30 patients who underwent FTO were compared with 87 eyes of 87 age- and IOP-pair-matched patients who underwent conventional trab (control group) (Table 1). Both groups were also similar regarding sex, preoperative VA, proportion of topical anti-glaucomatous treatment, and previous ocular surgeries, except for cataract surgery. Significantly more patients with pigmentary glaucoma were included in the FTO group $(P=0.02)$. Twenty-nine patients $(96.7 \%)$ treated with FTO were prospectively followed for 12 months. One patient was lost to follow-up, thus providing no follow-up data to postoperative analyses. Of the 87 patients in the control group with conventional trab, follow-up data were available at the 12-month visit for $71(81.6 \%)$.

\section{Success}

Complete success rate - defined as IOP $<18 \mathrm{mmHg}$ and IOP reduction of $\geq 30 \%$ without treatment with anti-glaucomatous drugs as well as survival of success - was not statistically significant $\left(P_{\text {log-rank }}=0.25\right)$ between the groups within 12 months (Figure 2) or at any time point during follow-up (Table 2).

\section{Intraocular pressure}

The median preoperative IOP was $23.0 \mathrm{mmHg}$ in the trab group and the FTO group, respectively, and was significantly lower at any time point in both groups after surgery (all $P<0.001$, Figure 3). Overall, IOP values were similar in both groups for up to 12 months, however, significantly lower values were observed in the FTO group at Day $1(P=0.009)$

Table I Demographic data of both groups at baseline

\begin{tabular}{|c|c|c|c|}
\hline Demographic & $\begin{array}{l}\text { Conventional } \\
\text { trabeculectomy, } n=87\end{array}$ & $\begin{array}{l}\text { Modified filtering } \\
\text { trabeculotomy, } n=30\end{array}$ & $P$-value ${ }^{a}$ \\
\hline Eye & & & 0.8 \\
\hline Right & $46(52.9)$ & $15(50.0)$ & \\
\hline Left & $41(47.1)$ & $15(50.0)$ & \\
\hline Age, years & $66.9 \pm 9.2$ & $66.7 \pm 10.0$ & 0.9 \\
\hline Range & $46-88$ & $47-86$ & \\
\hline Sex & & & 0.3 \\
\hline Male & $43(49.4)$ & II (36.7) & \\
\hline Female & $44(50.6)$ & $19(63.3)$ & \\
\hline BCVA, logMAR & $0.10(0.00-0.22)$ & $0.05(0.00-0.22)$ & 0.6 \\
\hline $\mathrm{IOP}, \mathrm{mmHg}$ & $23.0(20.0-27.0)$ & $23.0(20.0-27.0)$ & 0.9 \\
\hline Treatment with topical glaucoma drugs & $86(98.9)$ & $30(100.0)$ & 1.0 \\
\hline Topical glaucoma medications, $\mathrm{n}$ & $3(2-3)$ & $3(3-4)$ & 0.04 \\
\hline Type of glaucoma & & & 0.02 \\
\hline POAG & $67(77.0)$ & $20(66.7)$ & \\
\hline PEXG & $19(21.8)$ & $6(20.0)$ & \\
\hline PG & I (I.2) & $4(13.3)$ & \\
\hline Previous ocular surgery & & & 0.03 \\
\hline None & $50(57.5)$ & 14 (46.7) & \\
\hline Laser trabeculoplasty & $29(33.3)$ & $10(30.0)$ & \\
\hline Cyclodestructive & $8(9.2)$ & $0(0.0)$ & \\
\hline Laser peripheral iridotomy & $4(4.6)$ & $2(6.7)$ & \\
\hline Phaco & $9(10.3)$ & $9(30.0)$ & \\
\hline
\end{tabular}

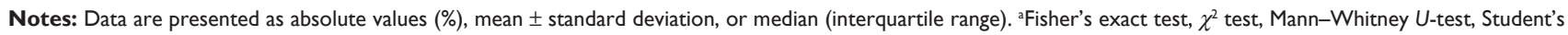
$t$-test, as appropriate. 'Laser peripheral iridotomy was performed in eyes with slightly narrow angles or pigmentary glaucoma.

Abbreviations: BCVA, best-corrected visual acuity; IOP, intraocular pressure; logMAR, logarithm of the minimum angle of resolution; PEXG, pseudo-exfoliative glaucoma; PG, pigmentary glaucoma; phaco, phacoemulsification; POAG, primary open-angle glaucoma. 


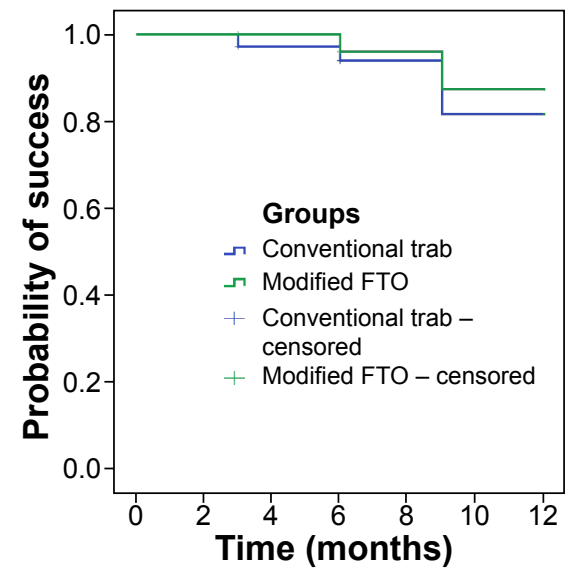

Figure 2 Kaplan-Meier survival curve of complete success. Complete success was defined as an intraocular pressure (IOP) of $<18 \mathrm{mmHg}$ and IOP reduction of $30 \%$ or more without medication. Success rates were not significantly different $\left(P_{\log \text {-rank }}=0.25\right)$ between both groups during follow-up. One patient treated with modified filtering trabeculotomy (FTO) was lost to follow-up, therefore data were available for 29 patients $(96.7 \%)$ prospectively followed for 12 months. Of the 87 conventional trabeculectomy (trab) patients, data for $7 \mathrm{I}$ patients (8I.6\%) were available at the $\mathrm{I}$-year visit. Patients with a follow-up of $<12$ months were counted as success or failure until the time point of drop out and were marked as censored.

and 6 months $(P=0.04)$ postoperatively (Figure 3$)$. The level of preoperative IOP was not significantly associated with the level of IOP at 12 months in the FTO group ( $\beta$-coefficient 0.002 [95\% confidence interval $-0.23 ; 0.24]$ ) or the trab group $(-0.06[-0.19 ; 0.06])$ (Figure 4A).

\section{Anti-glaucoma medication}

At baseline, patients in the FTO and trab groups received a median of three classes of topical glaucoma medications. Up to 3 months post-surgery, no patient was on medication in both groups. While at the 6-month time point, two trab patients $(2.6 \%)$ and no FTO-group patient were on topical drugs, one patient $(1.4 \%)$ in the trab group and two patients $(6.9 \%)$ in the FTO group needed additional IOP-lowering medications after 12 months.

\section{Visual acuity}

The median preoperative VA was 0.10 and $0.05 \log$ MAR in the trab and FTO groups, respectively $(P=0.6)$. Postoperative
VA was slightly reduced from preoperative values at 12 months (Figure 4B). The median VA was $0.16 \log$ MAR (IQR 0.05-0.30) in the trab group and 0.10 logMAR $(0.00-0.26)$ in the FTO group at the 12-month visit. However, the association of preoperative VA with values at 12 months differed between the trab and FTO groups $(P$-value for interaction $<0.01)$, indicating superior postoperative VA in the FTO group compared with in the conventional trab group.

\section{Postoperative complications and further surgical interventions}

Overall, postoperative complications and interventions were comparable in both groups (Table 3). Early postoperative complications, including elevated IOP (34.5\%) and hyphema $(48.3 \%)$ as a typical result of the trabeculotomy with FTO, were seen more frequently in the FTO group $(P<0.01)$. Therefore, laser suture lysis and scleral flap revision due to increased IOP were performed more often in the FTO group $(P=0.03)$. Scarring of the filtering bleb $(17.2 \%$ vs $13.8 \%)$ and consecutive bleb needling (17.2\% vs $13.8 \%)$ were observed more often in the conventional trab group $(P>0.05)$. In contrast to the conventional trab group, neodymium-doped yttrium aluminum garnet (Nd:YAG) laser goniopuncture was performed in three eyes $(10.3 \%)$ of the FTO group to increase aqueous flow through the fistula. During the late postoperative phase ( $>90$ days), postsurgical complications such as bleb scarring and consecutive increase of IOP were infrequent. In one patient $(3.4 \%)$ in the FTO group, an iris reposition had to be performed due to iris incarceration after Nd:YAG goniopuncture. No blebitis or endophthalmitis was seen after either procedure.

Additional IOP-lowering medication was added in case of insufficiently controlled IOP in both procedures, if scleral flap revision, goniopuncture, and bleb needling were not successful. None of the patients in either group received further glaucoma surgery in the early and late postoperative periods.

Table 2 Complete success ${ }^{\mathrm{a}}$

\begin{tabular}{lllll}
\hline $\begin{array}{l}\text { Follow-up, } \\
\text { months }\end{array}$ & $\begin{array}{l}\text { Conventional } \\
\text { trabeculectomy }\end{array}$ & $\begin{array}{l}\text { Modified filtering } \\
\text { trabeculotomy }\end{array}$ & \multicolumn{2}{l}{ OR (95\% CI) } \\
\hline 1 & $66 / 87(75.9)$ & $24 / 28(85.7)$ & Crude & Conditional $^{\mathrm{b}}$ \\
3 & $66 / 79(83.5)$ & $23 / 27(85.2)$ & $1.9 \mathrm{I}(0.59-6.13)$ & $2.5 \mathrm{I}(0.59-15.4)$ \\
6 & $59 / 76(77.6)$ & $22 / 24(91.7)$ & $1.13(0.34-3.83)$ & $1.23(0.28-7.56)$ \\
12 & $59 / 71(83.1)$ & $23 / 29(79.3)$ & $2.02(0.54-7.59)$ & $2.65(0.48-28.3)$ \\
\hline
\end{tabular}

Notes: Data are number of patients with complete success/number of patients examined at follow-up (proportion of success at follow-up). ${ }^{\mathrm{a}} \mathrm{Complete} \mathrm{success:} 1 \mathrm{OP}<18 \mathrm{mmHg}$ and $30 \%$ IOP reduction without glaucoma medication. ${ }^{\mathrm{b}} \mathrm{C}$ onditional logistic regression, accounting for pair-matching. Abbreviations: $\mathrm{Cl}$, confidence interval; IOP, intraocular pressure; OR, odds ratio. 


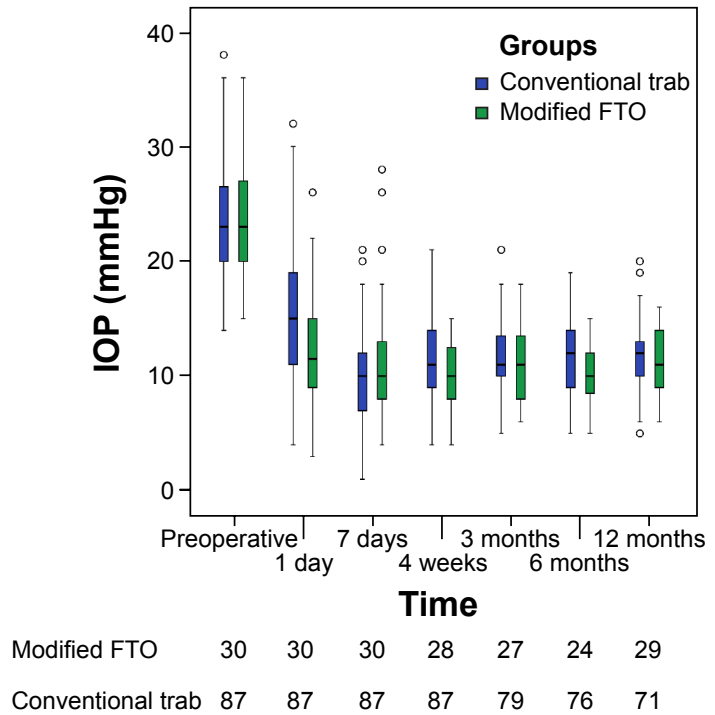

Figure 3 Intraocular pressure (IOP) results over 12 months of follow-up. IOP was significantly reduced in both groups during follow-up $(P<0.00 \mathrm{I})$. IOP was significantly lower in the modified filtering trabeculotomy (FTO) group than in the conventional trabeculectomy (trab) group at I day and 6 months after surgery. No statistical differences between groups were found for the remaining time points. One patient treated with FTO was lost to follow-up, therefore the data for 29 patients (96.7\%) were prospectively followed for 12 months. Of the 87 trab patients, data for 71 patients $(81.6 \%)$ were available at the I-year visit.

Notes: The black line in the center of each box plot represents the median, the boxes represent the 25th and 75th percentiles, the upper and lower bars are the 1.5 interquartile ranges, and the circles are outliers.

\section{Discussion}

Since its introduction by Cairns in 1968, trab remains the most commonly performed glaucoma surgery to effectively reduce IOP. ${ }^{1}$ So far, numerous surgical ${ }^{2,4-12}$ and

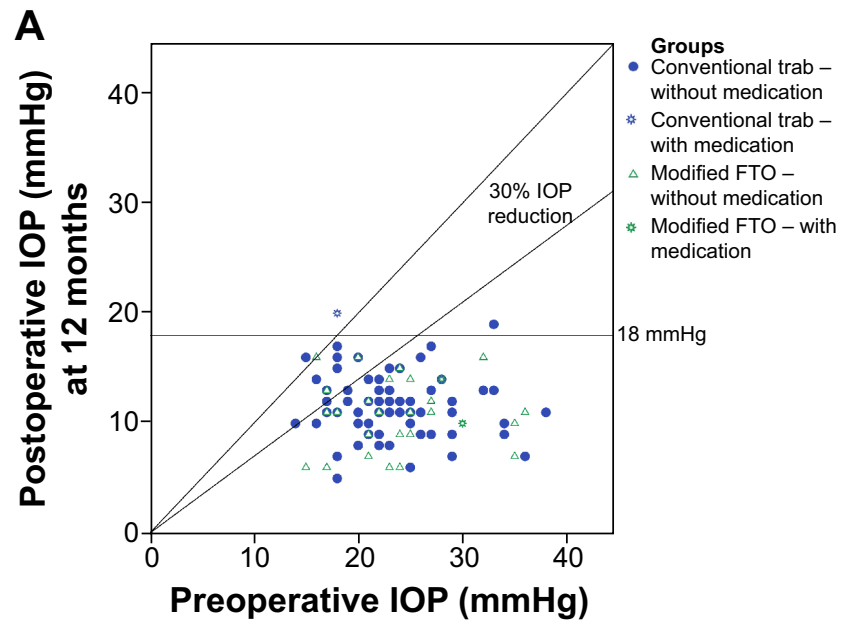

postsurgical ${ }^{13,14}$ variations have been described to improve its efficacy and safety while minimizing the risk of severe complications associated with filtering blebs. As glaucoma surgeons search for improvements to traditional filtering surgery, several modifications and new techniques of trab ${ }^{15-23}$ have gained growing interest in clinical research. Moreover, a combination of a non-penetrating surgical approach and trab has previously been described - the combination of trab, trabeculotomy, and sinusotomy, aiming to lower IOP while reducing the risk of hypotony-related complications. ${ }^{27,28}$ The intent was to combine the internalization with externalization of Schlemm's canal, providing a greater IOP reduction than trabeculotomy alone. In general, filtration surgery has been proven to result in a lower and more sustained IOP reduction than non-penetrating surgery. ${ }^{33,34}$

The results from our study suggest that this modified FTO without iridectomy provides adequate IOP control without glaucoma medication (complete success) comparable to conventional trab. The method described here includes steps from non-penetrating surgical techniques but purposely aims at reducing IOP using a guarded subconjunctival filtration. The architecture of the filtration site creates a two-level outflow resistance, thus reducing aqueous flow velocity to a more physiological rate. This may also have a favorable influence on wound healing or inflammation and additionally avoids the need for an iridectomy. VA was superior in the modified FTO group, possibly as a result of less anterior chamber inflammation or cataract progression. This might

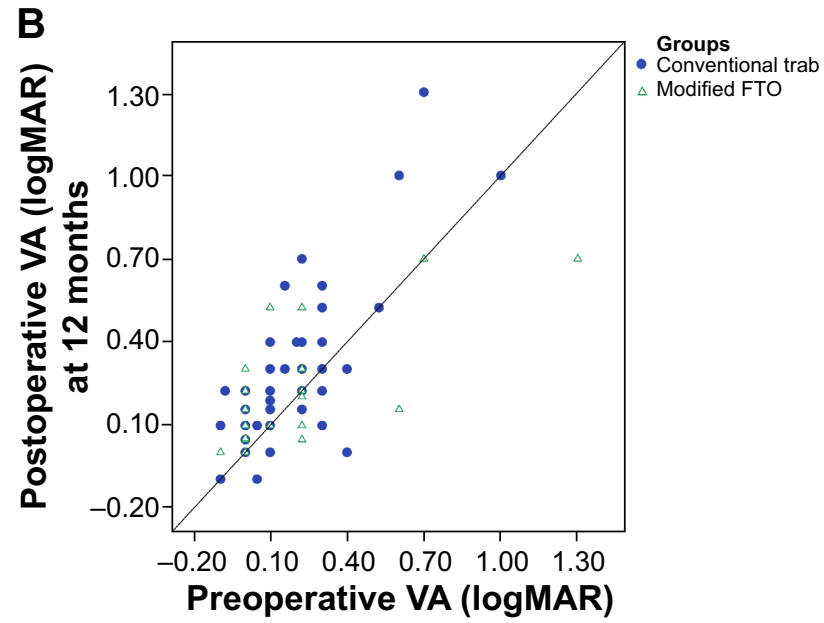

Figure 4 Scatter plots of $(\mathbf{A})$ intraocular pressure (IOP) and (B) visual acuity (VA) results compared with preoperative values for both groups. (A) Eyes below the line of $18 \mathrm{mmHg}$ and $30 \%$ IOP reduction fulfilled both criteria of success with or without medication (qualified success). (B) The association of preoperative VA with values at 12 months differed between the trabeculectomy (trab) and modified filtering trabeculotomy (FTO) groups ( $P$-value for interaction $<0.0 \mathrm{I})$. Postoperative $\mathrm{VA}$ was significantly better in patients undergoing FTO.

Notes: A single circle or triangle represents one eye with preoperative and postoperative IOP and VA at 12 months, respectively. The oblique line indicates no change of IOP or VA. Circles or triangles above the oblique line define a higher postoperative IOP or decrease in VA. 
Table 3 Postoperative complications and interventions

\begin{tabular}{|c|c|c|c|}
\hline Complication/intervention & $\begin{array}{l}\text { Conventional } \\
\text { trabeculectomy, } n=87\end{array}$ & $\begin{array}{l}\text { Modified filtering } \\
\text { trabeculotomy, n=29 }\end{array}$ & $P$-value \\
\hline \multicolumn{4}{|l|}{ Early complication ( $\leq 90$ days) } \\
\hline Elevated IOP (IOP > 25 mmHg) & $9(10.3)$ & $10(34.5)$ & 0.006 \\
\hline Hypotony (IOP <5 mmHg) & $18(20.7)$ & $7(24.1)$ & 0.8 \\
\hline Choroidal detachment & $2(2.3)$ & $3(10.3)$ & 0.2 \\
\hline Shallow anterior chamber & $3(3.4)$ & $0(0.0)$ & 0.7 \\
\hline Conjunctival leakage & $5(5.7)$ & I (3.4) & 0.8 \\
\hline Hyphema ( $\geq$ I mm layered blood) & $4(4.6)$ & $14(48.3)$ & $<0.001$ \\
\hline Iris incarceration & $2(2.3)$ & $0(0.0)$ & 1.0 \\
\hline Bleb scar & $15(17.2)$ & $4(13.8)$ & 0.9 \\
\hline \multicolumn{4}{|l|}{ Early intervention } \\
\hline Laser suture lysis, eyes & $4 I(47.1)$ & $21(72.4)$ & 0.03 \\
\hline Number of sutures, median (IQR) & I (I-2) & $2(1.5-3.5)$ & $<0.001$ \\
\hline 5-FU bleb injections, eyes & $57(65.5)$ & $18(62.1)$ & 0.9 \\
\hline Number of injections, median (IQR) & $5(3-7)$ & $5.5(3-6)$ & 0.7 \\
\hline Bleb needling & $15(17.2)$ & $4(13.8)$ & 0.9 \\
\hline Scleral flap revision (overfiltration) & $9(10.3)$ & $4(13.8)$ & 0.8 \\
\hline Scleral flap revision (elevated IOP) & $0(0.0)$ & $4(13.8)$ & 0.01 \\
\hline Conjunctival suture & $2(2.3)$ & $0(0.0)$ & 1.0 \\
\hline Iris reposition & $2(2.3)$ & $0(0.0)$ & 1.0 \\
\hline Nd:YAG laser goniopuncture & - & $3(10.3)$ & - \\
\hline \multicolumn{4}{|l|}{ Late complication ( $>90$ days) } \\
\hline Elevated IOP (IOP > 25 mmHg) & $0(0.0)$ & I (3.4) & 0.7 \\
\hline Hypotony (IOP $<5$ mmHg) & $0(0.0)$ & $2(6.9)$ & 0.1 \\
\hline Conjunctival leakage & $\mathrm{I}(\mathrm{I} .3)$ & $0(0.0)$ & 1.0 \\
\hline Iris incarceration & $0(0.0)$ & I (3.4) & 0.7 \\
\hline Bleb scar & $5(6.4)$ & $3(10.3)$ & 0.4 \\
\hline \multicolumn{4}{|l|}{ Late intervention } \\
\hline Bleb needling & $5(6.4)$ & $3(10.3)$ & 0.4 \\
\hline Iris reposition & $0(0.0)$ & I (3.4) & 0.7 \\
\hline Nd:YAG laser goniopuncture & - & $2(6.9)$ & - \\
\hline
\end{tabular}

Notes: Data are absolute values (\%) or median (interquartile range), as stated. a $P$-values derived from conditional logistic or linear regression, as appropriate.

Abbreviations: 5-FU, 5-fluorouracil; IOP, intraocular pressure; IQR, interquartile range; Nd:YAG, neodymium-doped yttrium aluminum garnet.

be an advantage over conventional trab, but needs to be confirmed in prospective, randomized trials.

Possible advantages of the modified filtration procedure over conventional trab are:

- stepwise controlled filtration: first, trabeculotomy results in a reduction of outflow resistance through the opened ostia of Schlemm's canal while avoiding the bulk flow of aqueous humor created by block excision or punch trab. Second, filtration through the scleral flap as a second level of resistance allows for further adjusting of the outflow rate. Both levels of resistance achieve a stepwise and gentler filtration control and prevent the IOP from falling abruptly in an all-or-nothing fashion. Gentle IOP reduction may reduce the risk of early postoperative hypotony and hypotony-related complications such as choroidal detachment, shallow anterior chamber, or hypotensive maculopathy leading to insufficient visual recovery or even loss of visual field.

- avoiding PI: as the trabeculo-Descemet membrane at the site of the deep scleral excision is preserved, no prolapse of the iris occurs, as it can during trab. It is therefore possible to avoid PI. This may be an advantage, as postoperative cataract progression and anterior chamber inflammation may be reduced. ${ }^{24,25}$

For this first series of 30 eyes, the side effects of FTO in our study are still similar to conventional trab, but include the hazards of the learning curve with this technique. In particular, a moderate hyphema often occurs in trabeculotomy due to reflux of blood from Schlemm's canal. This usually resorbs within days but may have caused transient IOP elevation. The occurrence of choroidal detachments was uncommon the following series of FTO operations and 
may be due to the learning curve. There was no statistically significant difference to trab.

An obvious difference of FTO from trab is the appearance of shallow diffuse blebs in FTO. However, this was not quantified by a bleb grading system in this study. For example, in FTO, massage does not result in a sudden subconjunctival ballooning of the bleb as it does in conventional trab. This is a sign of dampened outflow by the two resistance levels.

Recently, Eslami et al ${ }^{15}$ published a modified method of sutureless tunnel trab without performing a PI to reduce intraoperative manipulation through the small scleral incision and iridectomy-related complications such as bleeding, inflammation, and cataract progression. They have shown that sutureless tunnel trab without PI is as effective in controlling IOP as it is with PI and reduces the rate of bleeding and excessive inflammation. De Barros et $\mathrm{al}^{26}$ also concluded that trab performed without PI appeared to be as safe and effective at lowering IOP as when it is performed with PI. In our study of a modified FTO without PI, we found less bleb scarring, possibly due to less anterior chamber inflammation in the early postoperative period. Although a PI aimed at reducing the risk of an iris prolapse, no early iris incarceration occurred in the modified filtration surgery group without PI. One patient needed the reposition of an incarcerated iris after laser goniopuncture was performed during the late postoperative phase.

Stalmans et $\mathrm{al}^{20}$ reported their long-term results of a "safe" trab technique using releasable and adjustable sutures ${ }^{12}$ to allow a titration of outflow, a punch ${ }^{18}$ to perform a standard trab, and an anterior chamber maintainer during surgery to prevent intraoperative hypotony. This novel technique resulted in a stable IOP reduction over time with the minimum of postoperative complications, and offered the possibility of gentle adjustment of the IOP postoperatively. ${ }^{20}$ Although we did not use releasable or adjustable flap sutures to adjust IOP in our series, our technique achieved a guarded IOP control by reducing flow resistance step by step at two levels to prevent hypotony and related complications. The avoidance of PI in our technique may reduce inflammation with consecutive bleb failure and bleeding. Another advantage might be the more diffuse and less cystic filtering blebs of our new technique, which may reduce the risk of blebitis and endophthalmitis.

Mizoguchi et $\mathrm{al}^{27}$ described the results of combined trabeculotomy and sinusotomy compared with trab alone and confirmed that postoperative IOP levels after combined trabeculotomy and sinusotomy were lower in patients undergoing the modified trabeculotomy-sinusotomy procedure. Filtering blebs were found shortly after surgery, suggesting filtration after the combined method. However, blebs subsequently disappeared during further follow-up in most cases. The surgical technique described by Mizoguchi et $\mathrm{al}^{27}$ has similarities to our technique. However, in contrast to our technique of creating two scleral flaps, only one $4 \times 4 \mathrm{~mm}$ fornix-based scleral flap of $4 / 5$ thickness was made to expose Schlemm's canal and no MMC was used to reduce postoperative scarring of the filtering bleb. Additionally, Ogawa et $\mathrm{al}^{28}$ described a retrospective comparison of non-penetrating trab with sinusotomy with and without trabeculotomy and found a significantly higher IOP reduction and less medication use in eyes with an additional trabeculotomy. This technique resembles our FTO in creating two scleral flaps to unroof Schlemm's canal and performing trabeculotomy. However, a partial extraction of the outer scleral flap was done (so-called sinusotomy) which created a direct outflow from the deep sclerectomy lake. They did not compare the improved nonpenetrating trab and trabeculotomy with conventional trab and the control group was not matched for IOP. Additionally, no MMC was used. It is difficult to compare the study conducted by Ogawa et $\mathrm{al}^{28}$ with ours as they defined success as $\leq 21$ $\mathrm{mmHg}$ and only reported qualified success (with or without medication). In addition, the mean preoperative IOP was 21.0 $\mathrm{mmHg}$ in one group and $22.3 \mathrm{mmHg}$ in the other group, being very close to the success cut-off point.

\section{Limitations}

There are some limitations of the current study that need to be addressed. First, the total sample size and the number of patients treated with FTO in particular, were limited. However, we decided to study glaucoma patients in whom surgery was performed by a single physician, thus avoiding inter-individual variation among surgeons. Although the surgeon had considerable experience with conventional trab, trabeculotomy in children and adults, and non-penetrating surgery, the first 30 cases undergoing modified FTO were included during the surgeon's learning curve. The sample size is too small to comprehensively study the outcome and safety of the modified filtration procedure. To assess the latter, larger sample size and, in particular, longer follow-up data are needed to ensure sufficient numbers of rare outcomes and complications.

Second, while patients undergoing modified filtration surgery were followed prospectively, conventional trab patients were retrospectively identified and data were collected in part by chart review. While information bias is possible, study procedures and measurements were standard of care and part of clinical routine. 
Finally, the current study was not a randomized controlled trial. Due to the limited number of cases treated with modified filtration surgery, we selected controls by individualized pair-matching to reduce confounding by age and preoperative IOP.

\section{Conclusion}

A modification of filtering surgery without PI using twolevel resistance filtration was associated with significantly reduced IOP and number of medications in patients with open-angle glaucoma during a 1-year follow-up comparable to conventional trab. Post-surgery VA was significantly better in patients undergoing modified filtration surgery. However, future randomized and prospective studies with larger sample sizes are needed to confirm these results and to compare the efficacy and safety of this promising technique with conventional procedures.

\section{Acknowledgments}

This publication was funded by the German Research Foundation (DFG) and the University of Würzburg through the Open Access Publishing funding program.

\section{Disclosure}

Peter $U$ Heuschmann receives grants for research support from EU, BMBF, Charité, and BfArM. Franz Grehn receives funding for consultancy (Pharm Allergan and the European Glaucoma Advisory Board). Thomas Klink received travel grants for congress fees and accommodation (Novartis). None of the remaining authors has any conflicts of interest, including relevant financial interests, activities, relationships, or affiliations, to disclose related to this work. None of the remaining authors received funding for this work.

Results were presented at the Deutsche Ophthalmologische Gesellschaft Kongress, September 19-22, 2013, in Berlin, Germany.

\section{References}

1. Cairns JE. Trabeculectomy. Preliminary report of a new method. Am J Ophthalmol. 1968;66(4):673-679.

2. Khaw PT. Advances in glaucoma surgery: evolution of antimetabolite adjunctive therapy. J Glaucoma. 2001;10(5 Suppl 1):S81-S84.

3. Jampel HD, Solus JF, Tracey PA, et al. Outcomes and bleb-related complications of trabeculectomy. Ophthalmology. 2012;119(4):712-722.

4. Edmunds B, Thompson JR, Salmon JF, Wormald RP. The National Survey of Trabeculectomy. II. Variations in operative technique and outcome. Eye (Lond). 2001;15(Pt 4):441-448.

5. Jones E, Clarke J, Khaw PT. Recent advances in trabeculectomy technique. Curr Opin Ophthalmol. 2005;16(2):107-113.

6. Khaw PT, Chiang M, Shah P, Sii F, Lockwood A, Khalili A. Enhanced trabeculectomy: the Moorfields Safer Surgery System. Dev Ophthalmol. 2012;50:1-28.
7. Wells AP, Cordeiro MF, Bunce C, Khaw PT. Cystic bleb formation and related complications in limbus-versus fornix-based conjunctival flaps in pediatric and young adult trabeculectomy with mitomycin C. Ophthalmology. 2003;110(11):2192-2197.

8. Birchall W, Wakely L, Wells AP. The influence of scleral flap position and dimensions on intraocular pressure control in experimental trabeculectomy. J Glaucoma. 2006;15(4):286-290.

9. Kim YY, Sexton RM, Shin DH, et al. Outcomes of primary phakic trabeculectomies without versus with 0.5 - to 1 -minute versus 3 - to 5-minute mitomycin C. Am J Ophthalmol. 1998;126(6):755-762.

10. Matlach J, Panidou E, Grehn F, Klink T. Large-area versus small-area application of mitomycin $\mathrm{C}$ during trabeculectomy. Eur J Ophthalmol. 2013;23(5):670-677.

11. Aykan U, Bilge AH, Akin T, Certel I, Bayer A. Laser suture lysis or releasable sutures after trabeculectomy. J Glaucoma. 2007;16(2):240-245.

12. Wells AP, Bunce C, Khaw PT. Flap and suture manipulation after trabeculectomy with adjustable sutures: titration of flow and intraocular pressure in guarded filtration surgery. J Glaucoma. 2004;13(5): 400-406.

13. Khaw PT, Chang L, Wong TT, Mead A, Daniels JT, Cordeiro MF. Modulation of wound healing after glaucoma surgery. Curr Opin Ophthalmol. 2001;12(2):143-148.

14. Klink T, Grehn F. [Suture management after trabeculectomy.] Ophthalmologe. 2009;106(4):364-367. German.

15. Eslami Y, Mohammadi M, Khodaparast M, et al. Sutureless tunnel trabeculectomy without peripheral iridectomy: a new modification of the conventional trabeculectomy. Int Ophthalmol. 2012;32(5):449-454.

16. Gous PN, Roux P. Preliminary report of sutureless phacotrabeculectomy through a modified self-sealing scleral tunnel incision. J Cataract Refract Surg. 1995;21(2):160-169.

17. Lai JS, Lam DS. Trabeculectomy using a sutureless scleral tunnel technique: a preliminary study. J Glaucoma. 1999;8(3):188-192.

18. Zohdy GA, Lukaris A, Rogers ZA, Hill A, Roberts-Harry TJ. Early results of punch trabeculectomy. Int Ophthalmol. 1998;22(4):253-256.

19. Ophir A. Mini-trabeculectomy as initial surgery for medically uncontrolled glaucoma. Am J Ophthalmol. 2001;132(2):229-234.

20. Stalmans I, Gillis A, Lafaut AS, Zeyen T. Safe trabeculectomy technique: long term outcome. Br J Ophthalmol. 2006;90(1):44-47.

21. Buys JM. Trabeculectomy with ExPRESS: weighing the benefits and cost. Curr Opin Ophthalmol. 2013;24(2):111-118.

22. Yablonski ME. Trabeculectomy with internal tube shunt: a novel glaucoma surgery. J Glaucoma. 2005;14(2):91-97.

23. Skalicky SE, Lew HR. Surgical outcomes of combined trabeculectomycyclodialysis for glaucoma. J Glaucoma. 2015;24(1):37-44.

24. Rulli E, Biagioli E, Riva I, et al. Efficacy and safety of trabeculectomy vs nonpenetrating surgical procedures: a systematic review and metaanalysis. JAMA Ophthalmol. 2013;131(12):1573-1582.

25. Grehn F, Müller E. [Long term results following preventive iridectomy. A retrospective study.] Fortschr Ophthalmol. 1990;87(3):260-263. German.

26. De Barros DS, Da Silva RS, Siam GA, et al. Should an iridectomy be routinely performed as a part of trabeculectomy? Two surgeons' clinical experience. Eye (Lond). 2009;23(2):362-367.

27. Mizoguchi T, Nagata M, Matsumura M, Kuroda S, Terauchi H, Tanihara H. Surgical effects of combined trabeculotomy and sinusotomy compared to trabeculotomy alone. Acta Ophthalmol. 2000;78(2): 191-195.

28. Ogawa T, Dake Y, Saitoh AK, et al. Improved nonpenetrating trabeculectomy with trabeculotomy. J Glaucoma. 2001;10(5):429-435.

29. Klink T, Kann G, Ellinger P, Klink J, Grehn F, Guthoff R. The prognostic value of the wuerzburg bleb classification score for the outcome of trabeculectomy. Ophthalmologica. 2011;225(1):55-60.

30. Heuer DK, Barton K, Grehn F, Shaarawy T, Sherwood M. Consensus of definitions of success. In: Shaarawy T, Grehn F, Sherwood M, editors. Guidelines on Design and Reporting of Glaucoma Surgical Trials; World Glaucoma Association. Amsterdam: Kugler; 2008:15-24. Available from: www.worldglaucoma.org/Download/dl_files.php?id=1. Accessed January 22, 2015. 
31. Grehn F, Klink T. A new 6 o'clock traction suture technique for glaucoma filtration surgery. J Glaucoma. 2011;20(1):28-29.

32. Marquardt D, Lieb WE, Grehn F. Intensified postoperative care versus conventional follow-up: a retrospective long-term analysis of 177 trabeculectomies. Graefes Arch Clin Exp Ophthalmol. 2004;242(2):106-113.

33. Grehn F. Comparison of trabeculectomy with non-penetrating drainage glaucoma surgery in open-angle glaucoma. In: Weinreb RN, Crowston JG, editors. Glaucoma Surgery: Open-Angle Glaucoma. Consensus series 2. Amsterdam: Kugler; 2005:109-116.
34. Rulli E, Biagioli E, Riva I, et al. Efficacy and safety of trabeculectomy vs nonpenetrating surgical procedures. A systematic review and metaanalysis. JAMA Ophthalmol. 2013;131(12):1573-1582.

\section{Publish your work in this journal}

Clinical Ophthalmology is an international, peer-reviewed journal covering all subspecialties within ophthalmology. Key topics include: Optometry; Visual science; Pharmacology and drug therapy in eye diseases; Basic Sciences; Primary and Secondary eye care; Patient Safety and Quality of Care Improvements. This journal is indexed on

\section{Dovepress}

PubMed Central and CAS, and is the official journal of The Society of Clinical Ophthalmology (SCO). The manuscript management system is completely online and includes a very quick and fair peer-review system, which is all easy to use. Visit http://www.dovepress.com/ testimonials.php to read real quotes from published authors. 